\title{
OPENKI \\ The platform for open education
}

\author{
Urban Sand \\ Autonome Schule Zürich \\ Zürich, Switzerland \\ urban@xiala.net
}

\author{
Stephan Balmer \\ Denk:mal Bern \\ Bern, Switzerland \\ sb@cis.ch
}

\author{
Emre Sarigol \\ ETH Zürich \\ Zürich, Switzerland \\ semre@ethz.ch
}

\author{
Luca Obertüfer \\ Autonome Schule Frauenfeld \\ Zürich, Switzerland \\ lucaobertuefer@mailbox.org
}

\begin{abstract}
In this demo we aim to introduce and demonstrate OPENKI, an interactive web-platform designed and developed with the aim of facilitating a barrier-free access to education. In a nutshell, OPENKI is an open-source tool to facilitate selforganized, regionalized and offline knowledge exchange. It provides a basis for mediating non-commercial education opportunities by means of acting as a meeting point for individuals interested in similar subjects. The platform connects individuals who are interested learning or teaching specific topics, or have a physical space to offer where the coordinated events can take place, and gather themselves around interest groups. Through its lightweight interface, all stages of a learning processes are made possible from the selection of topics and the organization and execution of courses up to the documentation of the learning material.
\end{abstract}

\section{ACM Classification Keywords}

H.5.2 Information Interfaces and Presentation: User Interfaces;

\section{Author Keywords \\ Open Education; Social Network; Knowledge Exchange; Self Organization.}

\section{INTRODUCTION}

OPENKI ${ }^{1}$ is motivated by a set of existing needs in education and knowledge exchange. First, despite the growing variety of institutional- and private-sector education and advanced training offers, access to new knowledge is still short of being open for many individuals due mainly to socio-economic conditions where high costs, entrance limitations and language barriers prevail, hampering potential learners to access knowledge. Second, providers of advanced training possibilities for low access barrier are poorly connected and possess

\footnotetext{
1 http://openki.net

Copyright $\odot 2015$ is held by the author(s). Publication rights licensed to Aarhus University and ACM.

5th Decennial Aarhus Conference on Critical Alternatives August 17 - 21, 2015, Aarhus Denmark
}

DOI: http://dx.doi.org/10.7146/aahcc.v1i1.21319 only a limited capacity for the distribution and organization of what they have to offer, mainly due to centralization of teaching resources. The internet opens up vast possibilities for information exchange. However a platform fully dedicated to utilizing these in favor of education and knowledge exchange through a social framework is still missing. OPENKI aims to fill this gap by inheriting an alternative perspective which leans away from conventional education to a digitilized, regionalized and barrier-free education.

\section{TECHNOLOGY \& DEMO SETUP}

At the heart of it, OPENKI is built using Meteor, a Node.js based web application platform. The platform extends the Meteor framework through a set of libraries that provide community support where individuals may express membership to multiple interest groups and take different roles within these groups. Using a variety of roles in a peer-to-peer fashion, the platform gives individuals the freedom to organize or take part in events and courses, watch updates, physically host events, etc. The designed community framework is able to support features such as regionalization and formation of adhoc interest groups based on the specified interest. Through these features, each user views the piece of information that is most relevant to herself at all times, and is closer to the individuals with similar interests and in nearby regions. These features also improve server load optimization significantly

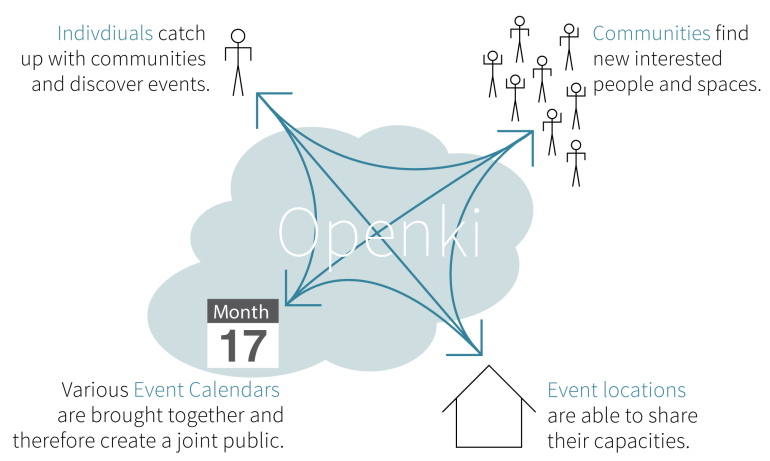

Figure 1. An overview of how users interact with OPENKI to announce interests and take part in relevant events. 


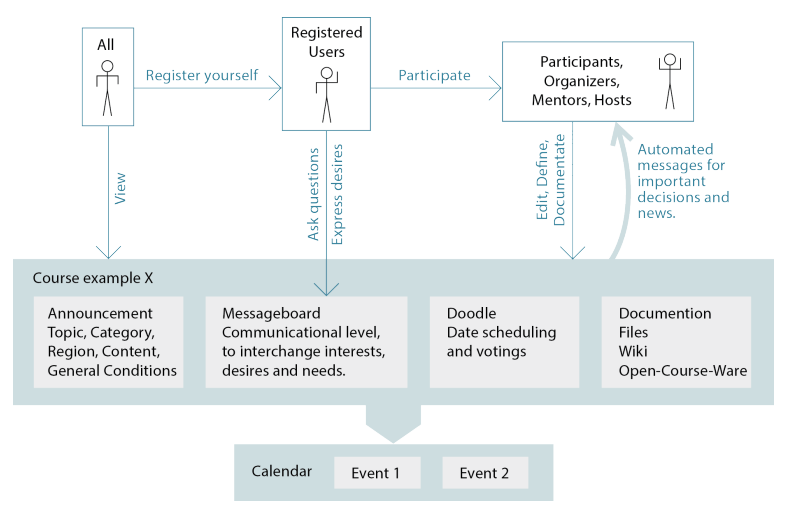

Figure 2. Workflow from course proposal to meet-up

since the information presented to the individual is filtered by region and interests.

Figure 1 summarizes how the platform provides interaction among individuals. As of the time of this writing, the platform is used by the Autonome Schule Zürich, an independent organization that aims at providing voluntary education for underprivilaged indivudals that reside in Zürich, Switzerland. What follows is some of the features that we would like to introduce in our demo.

\section{Selection of topics}

The learning contents are created by the users and are as diverse as their interests. They can range from language courses and theater workshops to outdoor survival camps, from beekeeping to training weeks in programming. Registered users can propose the course of interest in cases where a similar course does not exist. Proposed course announcements build the seeds of new courses that are to be organized. A subscription function informs interested users about changes within a certain subject.

\section{Organizing Courses}

OPENKI offers a series of tools and roles to coordinate and develop real courses from subject proposals. Specifically, four functional roles exist for each course: participant, organizer, mentor, host. Each individual can have have multiple roles among different courses. Once a course proposal attracts enough interested individuals (5 to 8 ), the course is bound to take place at a proposed time and space that the host can offer or suggest. Therefore each coordinated course is tied with a physical space. Therefore, the course organization scheme opens ways for more efficient usage of existing learning infrastructures. Course times are fixed using a voting tool (Doodle) and then added to an event calendar. For further decisions of all kinds, the platform offers the possibility to start a round of voting. Course participants are informed about any sort of change through a publish/subscribe mechanism using e-mails and SMS. Course-relevant documents (scripts, videos etc.) can be exchanged using the course organization interface. These documents can then be viewed and stored by all users. Finally, each course can be replicated for the weeks or months to come. This entire workflow is presented in Fig.2.

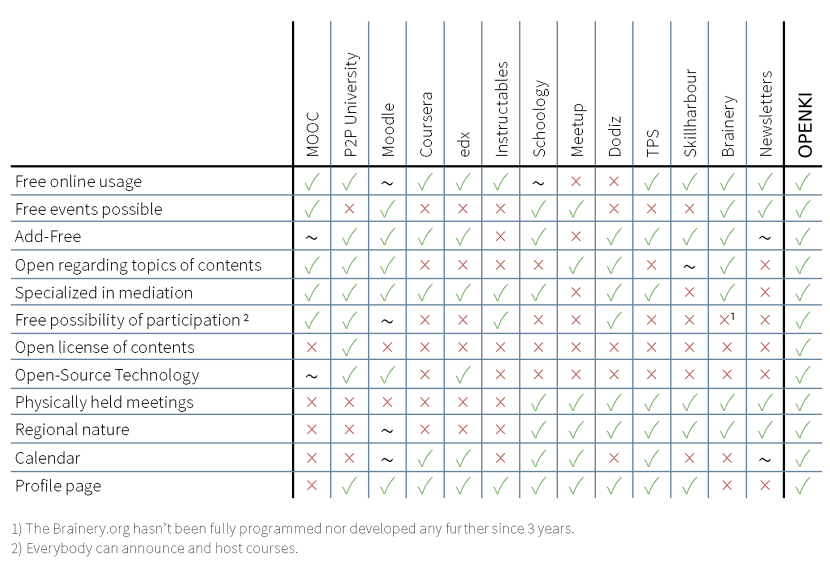

Figure 3. Comparison of existing platforms that offer open education

\section{COMPARISON TO EXISTING ONLINE-PLATFORMS}

The Internet and web technologies have a higher impact on education processes each year. In the past few years various online platforms and offerings that are committed to open education have emerged. However, existing platforms differ strongly in terms of mission, functionality and properties from OPENKI. We have analyzed existing platforms primarily according to the following three categories:

- Regionalization and physical meetings: A principal distinction exists between the so-called E-Learning Systems (only digital contents) and local based platforms, which render physical learning meet-ups possible.

- Financial access barriers: Through the usage of the website and the access to the actual knowledge contents, users may be subject to costs. On the contrary, OPENKI is accessible for free and distinguishes itself as an ad-free platform exclusively for non-commercial educational offers.

- Openness of contents: Fundamental criteria for content openness are: 1. Specification of topics, 2. Possibility for users to put up content, 3. An open license for the content, 4. Open-source technology. Diagram 3 compares some of the known platforms according to the mentioned dimensions. As a platform with a very low access barrier, OPENKI stands out as a unique choice in meeting all three criteria we have identified as necessary.

\section{CONCLUSION}

We have introduced OPENKI, an open source tool built using the Meteor framework, aimed at providing a social network for open education. We think that our platform differs in many ways from existing platforms for the reasons discussed above. Thanks to the platform's flexible design, we believe that many individuals can use and benefit from the platform to socialize with other individuals and have access to free and open education. This demo will be a good introduction point to make the platform known to a valuable community. 